\title{
Short-term stent coverage of second-generation zotarolimus-eluting durable polymer stents: Onyx one-month optical coherence tomography study
}

\author{
Tomasz Roleder ${ }^{1}$, Elvin Kedhi ${ }^{2}$, Balazs Berta², Pawel Gasior ${ }^{1}$, Wojciech Wanha ${ }^{1}$, Magda Roleder ${ }^{1}$, \\ Joanna Fluder ${ }^{1}$, Grzegorz Smolka ${ }^{1}$, Andrzej Ochala ${ }^{1}$, Wojciech Wojakowski ${ }^{1}$ \\ ${ }^{1}$ Department of Cardiology and Structural Heart Diseases, $3^{\text {rd }}$ Division of Cardiology, Medical University of Silesia, Katowice, Poland \\ ${ }^{2}$ Isala Haartcentrum, Zwolle, Netherlands
}

Adv Interv Cardiol 2019; 15, 2 (56): 143-150

DOI: https://doi.org/10.5114/aic.2019.86009

\begin{abstract}
Introduction: To date the early strut coverage with the second-generation durable-polymer ONYX zotarolimus-eluting stent (O-ZES) is unknown.

Aim: Optical coherence tomography (OCT) assessed the strut coverage of O-ZES at thirty-day follow-up.

Material and methods: OCT was performed after implantation and at 1-month follow-up in 15 patients treated with O-ZES.

Results: Mean patient age was $67 \pm 7$ years (73\% males). The clinical presentation consisted of acute coronary syndromes $(n=13)$ and stable coronary disease $(n=2)$. Four $(26 \%)$ patients had diabetes. OCT analysis was performed at baseline and 1-month follow-up in all stents. 378 cross-sections with 3582 struts were assessed at baseline and 3661 at follow-up. At follow-up, $88 \%$ struts were covered by tissue with a median thickness $37.91 \mu \mathrm{m}$ (IQR: 22.32-64.15). Median in-stent area obstruction by neointima was $2.64 \%$ (IQR: 1.70-4.84). From the total stent covered area, 92.3\% showed complete strut coverage. Homogeneous tissue was observed in $74 \%$ of cases. There were no differences in minimal lumen area $\left(5.07 \pm 1.08 \mathrm{~mm}^{2}\right.$ vs. $\left.4.81 \pm 0.94 \mathrm{~mm}^{2}, p=0.125\right)$ or minimal stent area $\left(4.95 \pm 1.22 \mathrm{~mm}^{2}\right.$ vs. $\left.4.92 \pm 0.99 \mathrm{~mm}^{2}\right)$ at baseline and at follow-up. There were no differences in the rate of strut malapposition ( $4.3 \%$ vs. $5.7 \%, p=0.417$ ). For all stents, malapposition volume was $47.9 \mathrm{~mm}^{3}$ at baseline and $51.7 \mathrm{~mm}^{3}$ at follow-up, giving the late acquired stent malapposition volume of $3.8 \mathrm{~mm}^{3}$.
\end{abstract}

Conclusions: The second-generation durable polymer O-ZES showed favorable vessel healing at 30-day OCT follow-up.

Key words: zotarolimus-eluting stent, vessel healing, optical coherence tomography, 1-month follow-up.

Su m m a ry

The present optical coherence tomography (OCT) study analyzed the coverage rate at 30 days after implantation of an ONYX zotarolimus-eluting stent (O-ZES). At 30 days, the coverage rate of the O-ZES was $88 \%$ with median thickness of $37.91 \mu \mathrm{m}$ (IQR: 22.32-64.15). The surface analysis showed that $92.3 \%$ of the lumen area had complete coverage of the stent. These findings suggest that the O-ZES has an excellent coverage rate at 30 days and therefore might be a stent platform which can be used in combination with short DAPT regimens for patients, especially those with a high risk of bleeding.

\section{Introduction}

Use of first-generation drug-eluting stents (DES) significantly reduced the restenosis rate after percutaneous coronary intervention $(\mathrm{PCl})$ compared to bare metal stents. However, this advantage was tempered by worse outcomes in safety endpoints. Patients treated with first-generation DES showed a higher incidence of stent thrombosis (ST), which was strongly correlated with poor outcomes such as myocardial infarction and death. Stent thrombosis can be related to incomplete re-endothelialization and vessel healing after stent implantation [1]. To avoid in-stent thrombosis, a prolonged dual antiplatelet therapy (DAPT) became mandatory [2]. Also, new generation devices were introduced with numerous improve-

Corresponding author:

Tomasz Roleder MD, PhD, Department of Cardiology and Structural Heart Diseases, $3^{\text {rd }}$ Division of Cardiology, Medical University of Silesia, Katowice, Poland, phone: +48 884096 034, e-mail: tomaszroleder@gmail.com

Received: 15.11.2018, accepted: 16.12.2018. 
ments in stent design. In particular, newer second-generation thin-strut devices covered with biocompatible, durable polymers resulted in significant reductions in ST compared to first-generation DES and bare-metal stent (BMS) [3]. While newer DES with biodegradable polymers were also introduced, the advantages of these devices with regard to safety outcomes as compared to first or second-generation DES are not so well proven [4]. Further improvements in stent engineering resulted in newer polymer-free DES. By eliminating the continuous peri-strut inflammation generated by durable polymers, these stents could theoretically provide faster vessel healing after stent implantation and behave as BMS after the drug was fully eluted. Indeed, the Leaders-Free trial showed that polymer-free biolimus-eluting stents were superior in efficacy and safety to BMS in high-risk bleeding patients undergoing $\mathrm{PCl}$ who received a short (1-month) DAPT duration [5]. However, direct comparison of this stent with a second-generation DES is missing. Furthermore, while strut coverage as measured by optical coherence tomography (OCT) is directly related to ST and consequently to DAPT duration, to date, we do not have evidence of coverage rates for this device at one month. Similarly, 1-month coverage rate data for second-generation DES are limited.

Since the time of drug elution from durable polymer and polymer free stents is almost the same, polymer presence should not impact the first 30 days of vessel healing $[6,7]$. Recently, a new zotarolimus-eluting stent covered with the durable BioLinx polymer (Resolute Onyx (O-ZES), Medtronic Santa Rosa, CA, USA) was introduced. Its platform is $10 \mu \mathrm{m}$ thinner as compared to the previous ZES (Resolute Integrity (R-ZES), Medtronic Santa Rosa, CA, USA). The thinner struts of the O-ZES stent may have the potential to induce faster vessel healing at one month compared to predicate stents.

\section{Aim}

Therefore, the present study assessed ONYX zotarolimus-eluting stent (O-ZES) healing by optical coherence tomography at 1-month follow-up.

\section{Material and methods}

The study was performed in patients undergoing a percutaneous coronary intervention with implantation of an O-ZES. The OCT imaging was performed after O-ZES implantation at baseline and 30 days follow-up. This study was carried out in the Upper-Silesian Medical Centre of the Medical University of Silesia in Katowice. The study conformed to the Declaration of Helsinki, and all patients gave written informed consent.

\section{Inclusion/exclusion criteria}

Patient aged 18 or older undergoing PCI with O-ZES for treatment in a native coronary artery and in a de novo lesion were enrolled in the study. After the initial angiogram, decisions about stent implantation were made according to the European Guidelines on Myocardial Revascularization [8].

Exclusion criteria were myocardial infarction (STEMI and NSTEMI), culprit lesions in coronary bypass grafts, in-stent restenosis, cardiogenic shock, left ventricular ejection fraction less than $20 \%$, significant valvular heart disease requiring surgery, contrast allergy, renal insufficiency with glomerular filtration rate less than $30 \mathrm{ml} /$ $\mathrm{min} / 1.73 \mathrm{~m}^{2}$, malignancy, and pregnancy. Finally, patients with tortuous lesions, ostial lesions or a vessel with reference diameter less than $2.00 \mathrm{~mm}$ or more than $3.5 \mathrm{~mm}$ were excluded from the study.

\section{The device}

The O-ZES stent has $81 \mu \mathrm{m}$ thin stent struts made from a composite wire of a cobalt alloy outer shell with a platinum-iridium inner core. The drug zotarolimus, a rapamycin derivative [9], is eluted using the durable BioLinx polymer, a proprietary blend of three polymers specifically designed for DES application enabling controlled and sustained drug release. Preclinical models have shown that the BioLinx polymer was associated with minimal inflammation which results in controlled neointimal hyperplasia and rapid, complete and functional endothelial healing $[10,11]$.

\section{Study procedure}

OCT imaging was performed after stent implantation and at 30 days. The ILUMIEN OPTIS system and Dragonfly catheters (Abbott, USA) were used for OCT imaging. The catheter was advanced through the stented segment over a 0.014 " coronary guidewire. The OCT probe (mid marker of the OCT catheter) was positioned $5 \mathrm{~mm}$ distal to the implanted stent intended for analysis. All OCT imaging was performed using automated pullback triggered by injection of contrast. All patients received unfractionated heparin before the OCT imaging to achieve an ACT of $>250$ s. OCT was used to optimize stent apposition after implantation as outlined in the CLI-OPCI II study [12]. After stent optimization, final OCT imaging was performed and was used for baseline assessment analysis. At thirty days, OCT imaging was performed in the same way.

\section{OCT analysis protocol}

All OCT pullbacks (frames) were initially screened for quality assessment and excluded from analysis if any portion of the image was out of the screen, a side branch occupied $>45^{\circ}$ of the cross-section, or the image had poor quality caused by residual blood, sew-up artifact, or reverberation. The strut-level analysis was performed considering all analyzable frames in 1-mm intervals along the entire region of interest (ROI). The ROI was estimated from the first distal and last proximal pull- 
back frame with the visible stent in more than $270^{\circ}$. The $5 \mathrm{~mm}$ vessel segments located proximally and distally to the ROI served as a reference segment. The reference vessel area was taken as the largest lumen area out of $5 \mathrm{~mm}$ long segments adjacent to the proximal or distal edge of the analyzed stent giving the proximal and distal reference area respectively. During the analysis, virtual contours of metallic struts were automatically drawn by the software. Its thickness was $87 \mu \mathrm{m}$ (strut thickness $=81 \mu \mathrm{m}+$ polymer coverage $=6 \mu \mathrm{m}$ ). The stent area was drawn for the endoluminal visible edge of the stent strut and ectoluminal edge of the virtually drawn stent contour: endoluminal stent area and out-stent area respectively. The lumen area was traced over the apposed struts and if present above its coverage. In the case of malapposed struts, the lumen area was traced over the vessel wall, adluminal to the malapposed strut. The stent struts were classified as well-apposed, malapposed, and non-apposed side branch (NASB), and as covered or uncovered. Strut malapposition was identified when there was a visible space between the virtually drawn strut and the lumen contour or if the distance between the strut blooming and lumen counter was more than $87 \mu \mathrm{m}$. If malapposition was visible, the malapposition area was drawn as a space between the lumen contour to the ectoluminal edge of the virtually drawn strut. The late acquired malapposition volume was measured as the difference between malapposition volume at baseline and at follow-up for all stents. The stent coverage was recognized when the thickness of the tissue covering the strut was $>0 \mu \mathrm{m}$. In order to measure the tissue thickness the distance from every metallic strut blooming to the lumen contour was measured. The out-stent neointima + stent strut area was estimated by subtraction of lumen area from the out-stent stent area. In the case of malapposition the out-stent neointima + stent strut area was measured as follows: the out-stent stent area - (lumen area malapposed area) [13]. The endoluminal neointimal area was measured as endoluminal stent area - lumen area. If the thickness of neointima between the visible strut did not exceed the endoluminal edge of the strut, the free neointima area was drawn, and the endoluminal neointima area was measured as stent area - (lumen area free neointima area). In-stent obstruction was measured as the (lumen area/endoluminal stent area) $\times 100$ and presented as a percentage (\%) [14]. Also, the total surface area of the lumen was measured in $1 \mathrm{~mm}$ steps to present the fraction of the vessel lumen covered by the uncovered stent. Characteristics of neointima which covered the struts were analyzed, and homogeneous and heterogeneous tissue was classified according to the previously published criteria $[15,16]$. The OCT analysis was performed by the independent Core Lab (Diagram, Zwolle, Netherlands) using the CAAS Intravascular 2.0 software (Pie Medical, Netherlands).

\section{Statistical analysis}

The Shapiro-Wilk test was used to analyze the continuous data distribution. Normally distributed values were presented as the mean \pm standard deviation. Non-normally distributed values were presented as the median with $25^{\text {th }}$ and $75^{\text {th }}$ percentile (interquartile range $-I Q R$ ). One-way ANOVA was used to compare normally distributed data, and the Mann-Whitney test was used to compare non-normally distributed data. For this analysis, a multilevel regression model was applied with random effects within the stent level, cross-section level and the strut level because of the clustering nature of data.

\section{Results}

Patients' characteristics and procedural details

Fifteen patients underwent $\mathrm{PCl}$ with implantation of 15 O-ZES in de novo lesions in native arteries. Mean patient age was $67 \pm 7$ years ( $73 \%$ males). The clinical presentation consisted of acute coronary syndrome $(n=13)$ and stable coronary disease $(n=2)$. Four $(26 \%)$ patients had diabetes.

All patients were treated with dual antiplatelet therapy during the entire duration of the study. Patients' characteristics are summarized in Table I.

The study stent was implanted in the following locations: 6 (40\%) in the left anterior descending artery, $4(27 \%)$ in the circumflex artery; $4(27 \%)$ in the right coronary artery and 1 (4\%) in the left main coronary artery. According to the ACC/AHA classification there were one A (6\%), four B1 (27\%), six B2 (40\%) and four C (27\%) lesions treated with O-ZES [17].

The median stent diameter and length were $3.0 \mathrm{~mm}$ (2.5-3.0) and $22 \mathrm{~mm}$ (18-26) respectively. One patient required bailout stenting with an everolimus-eluting stent (size $2.5 \times 11 \mathrm{~mm}$ ) to treat distal stent edge vessel dissection.

\section{OCT analysis}

OCT analysis was performed for all stents at the baseline procedure and at 1-month follow-up (30/-2 days) for a total of 378 cross-sections with 3582 struts assessed at baseline and 3661 struts at follow-up.

\section{ONYX stent analysis at baseline vs. 30-day follow-up}

There were no significant differences in minimal lumen area $\left(5.07 \pm 1.08 \mathrm{~mm}^{2}\right.$ vs. $4.81 \pm 0.94 \mathrm{~mm}^{2}, p=$ $0.125)$ or minimal stent area $\left(4.95 \pm 1.22 \mathrm{~mm}^{2}\right.$ vs. 4.92 $\left.\pm 0.99 \mathrm{~mm}^{2}, p=0.462\right)$ at baseline and at follow-up. There were no differences in the rate of stent strut malapposition (154 (4.3\%) vs. $234(5.7 \%), p=0.417)$ and in-stent malapposition volume $\left(\mathrm{mm}^{3}, 3.19 \pm 2.16\right.$ vs. $3.44 \pm 2.22$, $p=0.556)$ at baseline and follow-up. For all stents, the sum of malapposition volume was $47.9 \mathrm{~mm}^{3}$ at baseline 
and $51.7 \mathrm{~mm}^{3}$ at follow-up. Thus, the total late acquired stent malapposition volume was $3.8 \mathrm{~mm}^{3}$ (Figure 1).

\section{ONYX stent analysis at 30-day follow-up}

Percent area stenosis at follow-up was 17\% (5-27\%), with three stents having percent area stenosis more than 30\%, and the maximal in-stent obstruction (\%) equaled 2.64 (1.70-4.84). Ten (66\%) stents had at least one malapposed strut, and in all stents, at least one uncovered strut was identified. Nevertheless, the surface analysis showed that $534.88 \mathrm{~mm}^{2}$ (92\%) of total lumen surface area had covered stent struts (Table II, Figure 2).

Table I. Baseline demographic and clinical patient characteristics with implanted O-ZES $(n=15)$

\begin{tabular}{|c|c|}
\hline Parameter & Result \\
\hline Age [years] & $67 \pm 7$ \\
\hline Male gender, $n(\%)$ & $11(73)$ \\
\hline UA/SCAD, $n(\%)$ & $13(86) / 2(14)$ \\
\hline \multicolumn{2}{|l|}{ Risk factors, $n(\%)$ : } \\
\hline Hypertension & $13(86)$ \\
\hline Hyperlipidemia & $15(100)$ \\
\hline Diabetes mellitus & $4(26)$ \\
\hline Current smoking & $10(66)$ \\
\hline \multicolumn{2}{|l|}{ Pharmacological therapy, $n$ (\%): } \\
\hline Aspirin & $15(100)$ \\
\hline Clopidogrel & $11(73)$ \\
\hline Ticagrelor & $4(27)$ \\
\hline$\beta$-Adrenergic antagonist & $12(80)$ \\
\hline Calcium channel antagonist & $4(27)$ \\
\hline ARB/ACEI & $14(93)$ \\
\hline Statin & $14(93)$ \\
\hline Other lipid-lowering therapy & $3(20)$ \\
\hline Oral antidiabetics & $3(20)$ \\
\hline Insulin & $2(13)$ \\
\hline \multicolumn{2}{|l|}{ Laboratory results: } \\
\hline Total cholesterol, median (IQR) [mg/dl] & $145(131-179)$ \\
\hline LDL cholesterol [mg/dl] & $79(70-100)$ \\
\hline HDL cholesterol [mg/dl] & $44(39-53)$ \\
\hline Triglyceride [mg/dl] & $106(91-169)$ \\
\hline $\mathrm{GFR}$, mean $\pm \mathrm{SD}\left[\mathrm{ml} / \mathrm{min} / 1.73 \mathrm{~m}^{2}\right]$ & $79.06 \pm 17.58$ \\
\hline
\end{tabular}

$U A$ - unstable angina, SCAD - stable coronary artery disease, $A R B$ - angio tensin II receptor blocker, ACE - angiotensin-converting-enzyme inhibitor, $L D L$-low-density lipids, $H D L$ - high-density lipids, GFR - glomerular filtration rate.
In cross-section analysis, the median in-stent obstruction was $0.75 \%(0.12-2.33)$. There were 146 (43\%) cross-sections with at least one uncovered strut and 86 (22\%) with malapposition. Furthermore, 278 (74\%) cross-sections were covered with homogeneous neointima and 99 (26\%) with heterogeneous neointima (Table III).

The single strut analysis revealed that 486 (12\%) struts were uncovered, and 205 (5\%) struts were malapposed. $152(4.8 \%)$ malapposed struts were covered by the tissue. Furthermore, 68 (16.5\%) NASB struts were found, and 45 (1.1\%) were also covered by the neointima. The median neointimal thickness per single strut was 37.91 (22.32-64.15) $\mu \mathrm{m}$ (Table III, Figure 3).

\section{Discussion}

The present study is the first to date to show strut coverage rates of the novel O-ZES at 30 days. The main finding of this study is that the O-ZES had a very high rate of strut coverage at 30 days with $92 \%$ of the stent area consisting of covered struts. In addition, the study showed no stent recoil or significant late acquired malapposition at 30 days. Furthermore, the stent struts were covered predominantly with a homogeneous neointima. While this is the first study reported to date to evaluate O-ZES strut coverage with OCT, a previous OCT study of durable polymer R-ZES demonstrated similar results [18].

One-month OCT analysis demonstrated satisfactory O-ZES strut coverage by the neointima. The presented results are in line with $88 \%$ stent strut coverage for predicate R-ZES at 1-month follow-up, which has slightly thicker stent struts [18]. Furthermore, stent strut coverage was close to the value achieved for a polymer-free drug-filled stent (Medtronic Santa Rosa, CA, USA) in the RevElution study ( $\sim 88 \%$ vs. $\sim 91 \%$ ), and $\sim 92 \%$ of the lumen surface area was covered with stent struts [19]. Nevertheless, coverage is still less than the complete platform coverage observed in BMS [20]. As expected, the thickness of neointima was significantly lower as compared to BMS but nearly as thick as that observed for the drug-filled stent and for predicate R-ZES [18, 19]. It should be noted that tissue coverage was lower on malapposed struts and struts overlapping the bifurcation, confirming previously observed DES healing patterns [21], and supporting the notion that appropriate stent strut apposition is essential for fast vessel healing.

Compared to longer observations, the level of single O-ZES stent strut coverage and surface area coverage at 30 days was very close to that observed at 3-month follow-up for the R-ZES platform ( 94\%) [18, 22]. However, it should be noted that the O-ZES was implanted in a higher proportion of unstable patients as compared to the R-ZES study group ( $~ 85 \%$ vs. $\sim 30 \%$ ). It was higher than that observed at 2-month follow-up for the durable polymer platinum chromium everolimus-eluting stent ( $82 \%)$ [23], and CD34 sirolimus-eluting coronary stent 

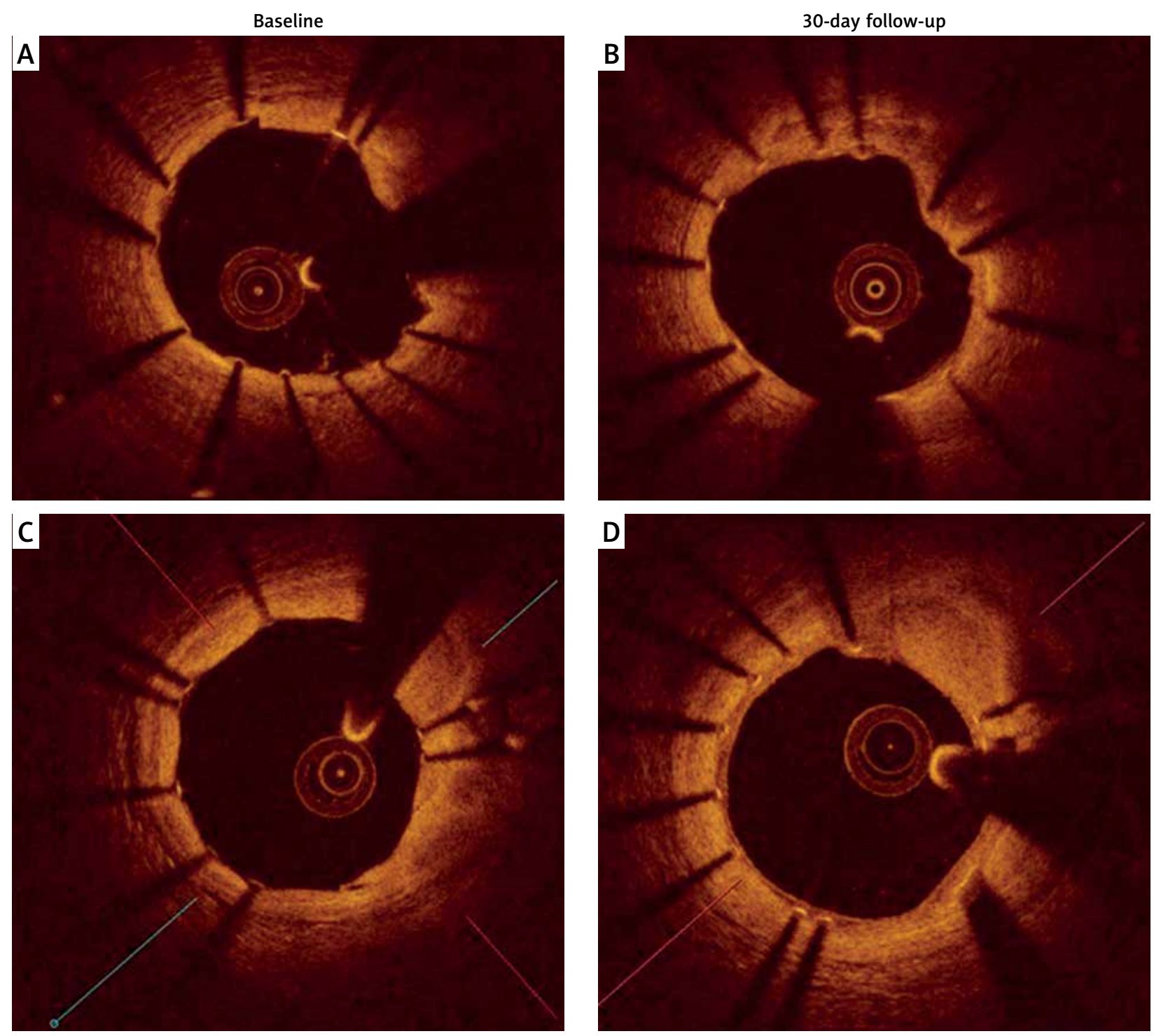

Figure 1. Representative OCT images of O-ZES coverage at 30-day follow-up. A, C - Baseline cross-sectional images of the implanted study stent. B - Cross-sectional image of study stent covered with homogeneous neointima. D - Cross-sectional image of study stent covered with heterogeneous neointima

( $85 \%)$ (COMBO stent, OrbusNeich, China) and for an open cell stent (Angiolite, iVascluar, Spain) at 3-month follow-up (86\%) [24, 25].

Not only the level of strut coverage is relevant for patients' outcome, but also the morphology of tissue which covers the struts. Seventy-four percent of the O-ZES stents were covered by homogeneous tissue, which was correlated in earlier reports with a high proportion of connective tissue and smooth muscle cells in histopathology, indicating favorable vessel healing [16]. Conversely, heterogeneous neointima was found to correlate with higher presence of fibrin as compared to homogeneous neointima [16] and was associated with poorer clinical outcomes [26]. Previous reports on durable and biodegradable polymer stents suggested that low-intensity neointima, identified at an early stage of stent healing, progressed to more mature homogeneous tissue within 6 months [27].

With the current study, OCT imaging was performed at baseline and one month after the stent implantation. The results showed that stent expansion and malposition did not change over such a short period, which confirmed stable stent geometry in vivo, despite a thinner strut design and a modified alloy composition.

Previous OCT studies documented that shortening DAPT to as short as 3 months did not impair vessel healing or clinical outcomes [28]. Given the satisfactory stent strut coverage at 1-month and following the data from the ZEUS study, which proved safety for one-month DAPT after zotarolimus-eluting Endeavor Sprint (Medtronic, Santa Rosa, CA, USA) stent implantation [29], that concept of short DAPT has a strong rationale and especially 
Table II. Stent level analysis of ONYX stent at 30-day follow-up

\begin{tabular}{|c|c|}
\hline Parameter & Result \\
\hline Stents, $n$ & 15 \\
\hline ROI length [mm] & $22(18-26)$ \\
\hline Proximal reference lumen area $\left[\mathrm{mm}^{2}\right]$ & $6.63 \pm 1.85$ \\
\hline Distal reference lumen area $\left[\mathrm{mm}^{2}\right]$ & $5.33 \pm 1.57$ \\
\hline Minimal lumen area $\left[\mathrm{mm}^{2}\right]$ & $4.81 \pm 0.94$ \\
\hline Endoluminal neointimal volume $\left[\mathrm{mm}^{3}\right]$ & $0.2(0-0.6)$ \\
\hline Out-stent neointimal volume $\left[\mathrm{mm}^{3}\right]$ & $39.79(35.56-43.56)$ \\
\hline Minimal out-stent area $\left[\mathrm{mm}^{2}\right]$ & $5.81 \pm 1.15$ \\
\hline Minimal endoluminal stent area $\left[\mathrm{mm}^{2}\right]$ & $4.81 \pm 1.05$ \\
\hline Malapposition volume $\left[\mathrm{mm}^{3}\right]$ & $3.4(1.65-4.60)$ \\
\hline$\%$ area stenosis & $17(5-27)$ \\
\hline $\begin{array}{l}\text { Stent with }>30 \% \text { area stenosis based } \\
\text { on ref. area }\end{array}$ & $3(20)$ \\
\hline Stent with > 1 malapposed strut, $n(\%)$ & $10(66)$ \\
\hline Stent with > 1 uncovered strut, $n(\%)$ & $15(100)$ \\
\hline Maximal in-stent obstruction (\%) & $2.64(1.70-4.84)$ \\
\hline Stent with > 1 intraluminal masses, $n(\%)$ & $1(6)$ \\
\hline Disrupted stent & 0 \\
\hline $\begin{array}{l}\text { Total lumen surface area analyzed } \\
\text { per } 1 \mathrm{~mm}\left[\mathrm{~mm}^{2}\right]\end{array}$ & 579,42 \\
\hline $\begin{array}{l}\text { Surface of lumen area covered by the } \\
\text { uncovered stent }\left[\mathrm{mm}^{2}\right], n(\%)\end{array}$ & $44.54(7.68)$ \\
\hline
\end{tabular}

for high-risk bleeding patients. Accordingly, the launched ONYX ONE trial will compare the safety of one-month DAPT after O-ZES implantation as compared to polymer free stent implantation (Clinical trials NTC 03344653).

\section{Study limitations}

There are several limitations of the study which need to be addressed. It was a single center study, and a small number of patients were enrolled. The study assessed only the O-ZES stent platform and did not provide data for a control group. The group of patients was heterogeneous including acute coronary patients, as well as diabetics ones. More even distribution of patients could provide deeper insight into the vessel healing regarding the presented pathology. On the other hand, such group diversity suggests that the study conclusions may be applied to broader clinical indications.

\section{Conclusions}

The second-generation durable-polymer O-ZES stent demonstrated a favorable vessel healing pattern at onemonth follow-up. These findings suggest that the O-ZES might be a stent platform which can be used in combi-

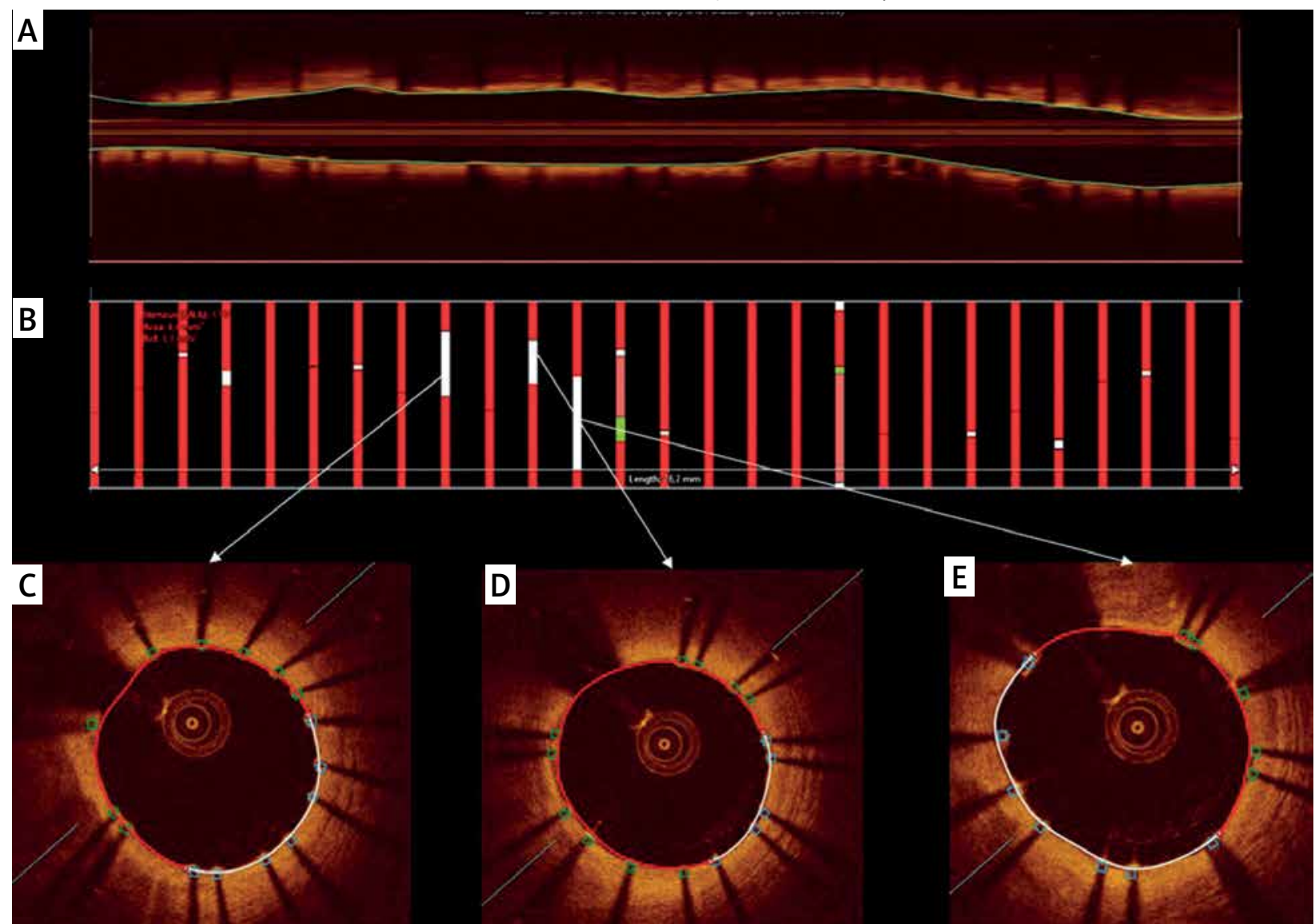

Figure 2. Representative images of O-ZES surface area analysis. A - Cross-sectional image of the implanted stent. B - Boxes indicate the $1 \mathrm{~mm}$ intervals of surface measurements. Red color indicates covered struts, other color indicates uncovered struts. C, D, E-Representative images of study stent with lumen surface (white line) covered with uncovered struts (blue boxes) 
Table III. Cross-sectional and strut-level analysis of ONYX stent at 30-day follow-up

\begin{tabular}{|c|c|}
\hline Variable & Result \\
\hline \multicolumn{2}{|l|}{ Cross-sectional analysis: } \\
\hline Cross-sections, $n$ & 378 \\
\hline Lumen area $\left[\mathrm{mm}^{2}\right]$ & $6.42 \pm 1.64$ \\
\hline Out-stent stent area $\left[\mathrm{mm}^{2}\right]$ & $7.57 \pm 1.42$ \\
\hline Endoluminal stent $\left[\mathrm{mm}^{2}\right]$ & $6.39 \pm 1.31$ \\
\hline Malapposition area $\left[\mathrm{mm}^{2}\right]$ & $0.60(0.36-0.88)$ \\
\hline Endoluminal neointimal area $\left[\mathrm{mm}^{2}\right]$ & $0.05(0.01-0.15)$ \\
\hline $\begin{array}{l}\text { Out-stent neointimal area }+ \text { stent strut } \\
\text { area }\left[\mathrm{mm}^{2}\right]\end{array}$ & $1.81(1.65-2.00)$ \\
\hline Cross-sections with intraluminal mass, $n(\%)$ & $5(1.3)$ \\
\hline Cross-sections with malapposition, $n(\%)$ & $86(22.5)$ \\
\hline Cross-sections with uncovered stents, $n(\%)$ & $146(43)$ \\
\hline In-stent obstruction (\%) & $0.75(0.15-2.33)$ \\
\hline $\begin{array}{l}\text { Homogeneous vs. heterogeneous } \\
\text { neointima, } n(\%)\end{array}$ & 278 (74) vs. 99 (26) \\
\hline \multicolumn{2}{|l|}{ Strut level analysis: } \\
\hline Stent struts, $n$ & 3661 \\
\hline Uncovered struts, $n(\%)$ & $450(12.3)$ \\
\hline Malapposed struts, $n$ (\%) & $205(5.7)$ \\
\hline Malapposed uncovered, $n$ (\%) & $53(1.45)$ \\
\hline NASB struts, $n(\%)$ & $68(1.85)$ \\
\hline NASB uncovered struts, $n(\%)$ & $23(0.62)$ \\
\hline Neointimal thickness per strut $[\mu \mathrm{m}]$ & $37.91(22.32-64.15)$ \\
\hline
\end{tabular}

$N A S B$ - non-apposed side branch.

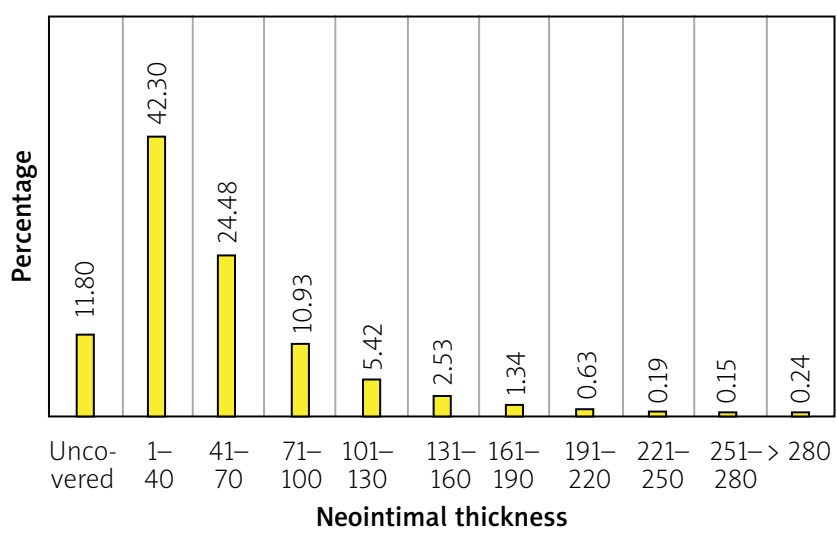

Figure 3. The proportion of neointimal thickness measured for a single strut

nation with short DAPT regimens for patients, especially with a high risk of bleeding. However, this hypothesis should be tested by large and randomized trials.

\section{Conflict of interest}

The authors declare no conflict of interest.

\section{References}

1. Finn $A V$, Nakazawa $G$, Joner $M$, et al. Vascular responses to drug eluting stents: importance of delayed healing. Arterioscler Thromb Vasc Biol 2007; 27: 1500-10.

2. Stettler C, Wandel S, Allemann S, et al. Outcomes associated with drug-eluting and bare-metal stents: a collaborative network meta-analysis. Lancet 2007; 370: 937-48.

3. Palmerini T, Benedetto U, Bacchi-Reggiani L, et al. Mortality in patients treated with extended duration dual antiplatelet therapy after drug-eluting stent implantation: a pairwise and Bayesian network meta-analysis of randomised trials. Lancet 2015; 385: 2371-82.

4. Zhang $\mathrm{Y}$, Tian N, Dong S, et al. Impact of biodegradable versus durable polymer drug-eluting stents on clinical outcomes in patients with coronary artery disease: a meta-analysis of 15 randomized trials. Chin Med J (Engl) 2014; 127: 2159-66.

5. Urban P, Meredith IT, Abizaid A, et al. Polymer-free drug-coated coronary stents in patients at high bleeding risk. N Engl J Med 2015; 373: 2038-47.

6. Udipi K, Melder RJ, Chen M, et al. The next generation Endeavor Resolute Stent: role of the BioLinx Polymer System. Eurolntervention 2007; 3: 137-9.

7. Tada N, Virmani R, Grant G, et al. Polymer-free biolimus a9-coated stent demonstrates more sustained intimal inhibition, improved healing, and reduced inflammation compared with a polymer-coated sirolimus-eluting cypher stent in a porcine model. Circ Cardiovasc Interv 2010; 3: 174-83.

8. Authors/Task Force $\mathrm{m}$, Windecker $\mathrm{S}$, Kolh P, et al. 2014 ESC/EACTS Guidelines on myocardial revascularization: The Task Force on Myocardial Revascularization of the European Society of Cardiology (ESC) and the European Association for Cardio-Thoracic Surgery (EACTS)Developed with the special contribution of the European Association of Percutaneous Cardiovascular Interventions (EAPCI). Eur Heart J 2014; 35: 2541-619.

9. Price MJ, Saito S, Shlofmitz RA, et al. First report of the Resolute Onyx 2.0-mm Zotarolimus-Eluting Stent for the treatment of coronary lesions with very small reference vessel diameter. JACC Cardiovasc Interv 2017; 10: 1381-8.

10. Udipi K, Chen M, Cheng P, et al. Development of a novel biocompatible polymer system for extended drug release in a next-generation drug-eluting stent. J Biomed Mater Res A 2008; 85: 1064-71.

11. Hezi-Yamit A, Sullivan C, Wong J, et al. Impact of polymer hydrophilicity on biocompatibility: implication for DES polymer design. J Biomed Mater Res A 2009; 90: 133-41.

12. Prati F, Romagnoli E, Burzotta F, et al. Clinical impact of OCT findings during PCl: the CLI-OPCI II Study. JACC Cardiovasc Imaging 2015; 8: 1297-305.

13. Nakatani S, Sotomi Y, Ishibashi Y, et al. Comparative analysis method of permanent metallic stents (XIENCE) and bioresorbable poly-L-lactic (PLLA) scaffolds (Absorb) on optical coherence tomography at baseline and follow-up. Eurolntervention 2016; 12: 1498-509.

14. Gomez-Lara J, Brugaletta S, Farooq V, et al. Head-to-head comparison of the neointimal response between metallic and bioresorbable everolimus-eluting scaffolds using optical coherence tomography. JACC Cardiovasc Interv 2011; 4: 1271-80.

15. Nagoshi R, Shinke T, Otake $H$, et al. Qualitative and quantitative assessment of stent restenosis by optical coherence tomography: comparison between drug-eluting and bare-metal stents. Circ J 2013; 77: 652-60. 
16. Kim JS, Afari ME, Ha J, et al. Neointimal patterns obtained by optical coherence tomography correlate with specific histological components and neointimal proliferation in a swine model of restenosis. Eur Heart J Cardiovasc Imaging 2014; 15: 292-8.

17. Myler RK, Shaw RE, Stertzer SH, et al. Lesion morphology and coronary angioplasty: current experience and analysis. J Am Coll Cardiol 1992; 19: 1641-52.

18. Hashikata T, Tojo T, Namba S, et al. Neointimal coverage of zotarolimus-eluting stent at 1, 2, and 3 months' follow-up: an optical coherence tomography study. Heart Vessels 2016; 31: 206-11.

19. Worthley SG, Abizaid A, Kirtane AJ, et al. First-in-human evaluation of a novel polymer-free drug-filled stent: angiographic, IVUS, OCT, and clinical outcomes from the revelution study. JACC Cardiovasc Interv 2017; 10: 147-56.

20. Prati F, Romagnoli E, Valgimigli $M$, et al. Randomized comparison between 3-month Cre8 DES vs. 1-month Vision/Multilink8 BMS neointimal coverage assessed by OCT evaluation: the DEMONSTRATE study. Int J Cardiol 2014; 176: 904-9.

21. Gomez-Lara J, Radu M, Brugaletta S, et al. Serial analysis of the malapposed and uncovered struts of the new generation of everolimus-eluting bioresorbable scaffold with optical coherence tomography. JACC Cardiovasc Interv 2011; 4: 992-1001.

22. Izumi D, Miyahara M, Fujimoto $N$, et al. Optical coherence tomography analysis of the stent strut and prediction of resolved strut malapposition at 3 months after 2nd-generation drug-eluting stent implantation. Heart Vessels 2016; 31: 1247-56.

23. Varho V, Kiviniemi TO, Nammas W, et al. Early vascular healing after titanium-nitride-oxide-coated stent versus platinum-chromium everolimus-eluting stent implantation in patients with acute coronary syndrome. Int J Cardiovasc Imaging 2016; 32: 1031-9.

24. Puri R, Otaegui I, Sabate M, et al. Three- and 6-month optical coherence tomographic surveillance following percutaneous coronary intervention with the Angiolite( $R)$ drug-eluting stent: the ANCHOR study. Catheter Cardiovasc Interv 2018; 91: 435-43.

25. Jaguszewski $M$, Aloysius R, Wang W, et al. The REMEDEE-OCT study: an evaluation of the bioengineered COMBO dual-therapy CD34 antibody-covered sirolimus-eluting coronary stent compared with a cobalt-chromium everolimus-eluting stent in patients with acute coronary syndromes: insights from optical coherence tomography imaging analysis. JACC Cardiovasc Interv 2017; 10: 489-99.

26. Kim JS, Lee JH, Shin DH, et al. Long-term outcomes of neointimal hyperplasia without neoatherosclerosis after drug-eluting stent implantation. JACC Cardiovasc Imaging 2014; 7: 788-95.

27. Legutko J, Gil RJ, Buszman PE, et al. An optical coherence tomography study of neointimal morphology and strut coverage at different time intervals from implantation of biodegradable polymer-coated sirolimus-eluting stents. Catheter Cardiovasc Interv 2018; 92: 302-9.

28. Fujimoto W, Sawada T, Toba T, et al. Comparison of the 9-month intra-stent conditions and 2-year clinical outcomes after resolute zotarolimus-eluting stent implantation between 3-month and standard dual antiplatelet therapy. J Cardiol 2018; 72: 66-73.

29. Valgimigli $M$, Patialiakas A, Thury A, et al. Zotarolimus-eluting versus bare-metal stents in uncertain drug-eluting stent candidates. J Am Coll Cardiol 2015; 65: 805-15. 